\title{
Potassium abundances in nearby metal-poor stars ${ }^{\star}$
}

\author{
H. W. Zhang ${ }^{1,2}$, T. Gehren ${ }^{2}$, K. Butler ${ }^{2}$, J. R. Shi ${ }^{2,3}$, and G. Zhao ${ }^{3}$ \\ 1 Department of Astronomy, School of Physics, Peking University, Beijing 100871, PR China \\ e-mail: zhw@bac.pku.edu.cn \\ 2 Institut für Astronomie und Astrophysik der Universität München, Scheinerstrasse 1, 81679 München, Germany \\ 3 National Astronomical Observatories, Chinese Academy of Sciences, Beijing 100012, PR China
}

Received 25 January 2006 / Accepted 16 June 2006

\section{ABSTRACT}

\begin{abstract}
Aims. The potassium abundances for 58 metal-poor stars are determined using high-resolution spectroscopy. The abundance trends in stars of different population are discussed.

Methods. All abundance results have been derived from NLTE statistical equilibrium calculations and spectrum synthesis methods. Results. The NLTE corrections are significant $(-0.20$ to $-0.55 \mathrm{dex})$ and they depend on the effective temperatures and surface gravities. The potassium abundances of thin disk, thick disk and halo stars show distinct trends, such as in the case of the $\alpha$-elements. $[\mathrm{K} / \mathrm{Fe}]$ gradually increases with a decrease in $[\mathrm{Fe} / \mathrm{H}]$ for thin disk stars, $[\mathrm{K} / \mathrm{Fe}]$ of thick disk stars is nearly constant at $[\mathrm{K} / \mathrm{Fe}] \sim$ $+0.30 \mathrm{dex}$; halo stars also have nearly constant values of $[\mathrm{K} / \mathrm{Fe}] \sim+0.20 \mathrm{dex}$.

Conclusions. The derived dependence between $[\mathrm{K} / \mathrm{Fe}]$ and $[\mathrm{Fe} / \mathrm{H}]$ is in agreement with the theoretical prediction of published model calculations of the chemical evolution of the Galaxy. The nearly constant $[\mathrm{K} / \mathrm{Mg}]$ ratio with small scatter suggests that the nucleosynthesis of potassium is closely coupled to the $\alpha$-elements.
\end{abstract}

Key words. line: formation - line: profiles - stars: abundances - stars: late-type - Galaxy: evolution

\section{Introduction}

The determination of the element abundances in stars of different metal abundances is important for understanding the chemical evolution of the Milky Way.

Potassium is produced mainly by explosive oxygen burning (Truran \& Arnett 1971; Woosley et al. 1973; Thielemann \& Arnett 1985; Woosley \& Weaver 1995). Theoretical studies of the Galactic evolution of chemical elements have given different predictions for the $[\mathrm{K} / \mathrm{Fe}]$ vs. $[\mathrm{Fe} / \mathrm{H}]$ dependence. Timmes et al. (1995) suggested a decreasing trend of $[\mathrm{K} / \mathrm{Fe}]$ with decreasing $[\mathrm{Fe} / \mathrm{H}]$ in a model with oxygen burning under hydrostatic equilibrium conditions. Goswami \& Prantzos (2000) showed a mildly supersolar behaviour of $[\mathrm{K} / \mathrm{Fe}] \simeq+0.2 \mathrm{dex}$ at $[\mathrm{Fe} / \mathrm{H}] \leq$ -1 . Samland (1998) considered a chemodynamical model for the synthesis of potassium, and he predicted that $[\mathrm{K} / \mathrm{Fe}]$ gradually increases from 0.0 at $[\mathrm{Fe} / \mathrm{H}] \simeq 0$ to +0.3 at $[\mathrm{Fe} / \mathrm{H}] \simeq-1.0$ and maintains a nearly constant value of $[\mathrm{K} / \mathrm{Fe}] \simeq+0.3$ at $-3 \leq$ $[\mathrm{Fe} / \mathrm{H}] \leq-1$ (see Fig. 5).

On the observational side, the spectroscopic determination of potassium abundances is difficult. Only the resonance doublet (K I 7665 and $7699 \AA$ ) is available for abundance analyses of metal-poor stars, however, $\mathrm{K}$ I resonance lines are often blended with very strong telluric $\mathrm{O}_{2}$ lines.

There have been only a few studies that attempted to investigate the stellar potassium abundance. Gratton \& Sneden (1987) first derived $\mathrm{K}$ abundances in stars of a large range of metal abundances. The results suggested that $[\mathrm{K} / \mathrm{Fe}]>0$ in metal-poor stars, as is the case for the $\alpha$-elements, but with large scatter at

\footnotetext{
* Based on observations collected at the German-Spanish Astronomical Center, Calar Alto, Spain.
}

a given $[\mathrm{Fe} / \mathrm{H}]$. Chen et al. (2000) showed a decreasing $[\mathrm{K} / \mathrm{Fe}]$ trend with increasing metal abundance for metal-poor disk stars. Tomkin et al. (1997) studied chemical abundances of 8 metalrich stars and found the same potassium abundance trend with metal abundance. Cayrel et al. (2004) presented potassium abundances for 35 very metal-poor giant stars with $[\mathrm{Fe} / \mathrm{H}]<-2.5$ and found that $[\mathrm{K} / \mathrm{Fe}]$ seems to decrease slowly with decreasing metal abundance. Zhang \& Zhao (2005) confirmed the supersolar $[\mathrm{K} / \mathrm{Fe}]$ abundance ratio in 32 halo stars.

Takeda et al. (1996, 2002) predicted large NLTE corrections for the potassium abundance analysis of the Sun and metal-poor stars, however their effect on the $[\mathrm{K} / \mathrm{Fe}]$ vs. $[\mathrm{Fe} / \mathrm{H}]$ relation is not large, since these corrections do not show any significant dependance on the metal abundance. Ivanova \& Shimansky (2000) and Shimansky et al. (2003) also found large NLTE corrections of the potassium abundance, and their $[\mathrm{K} / \mathrm{Fe}]_{\text {NLTE }}$ of disk stars decreases systematically from 0.3 to -0.1 when the stellar metal abundance increases from $[\mathrm{Fe} / \mathrm{H}]=-1.0$ to 0.2 ; this is in agrement with the results of Galactic chemical evolution models.

Zhang et al. (2006, hereafter Paper I) investigated the formation of neutral potassium lines in the solar photosphere based on extensive statistical equilibrium calculations, and obtained a solar potassium abundance of $\log \varepsilon_{\odot}(\mathrm{K})=5.12$, which is close to the meteoritic abundance of 5.12 (Grevesse \& Sauval 1998) or 5.09 (Lodders 2003).

In this paper we present potassium abundances for 58 metalpoor stars based on the NLTE model of Paper I. In Sect. 2, we describe the observational data. In Sect. 3, we introduce the atmospheric models and stellar parameters. In Sect. 4, we document the NLTE calculations. The discussion is presented in Sect. 5, and the conclusions are found in the last section. 


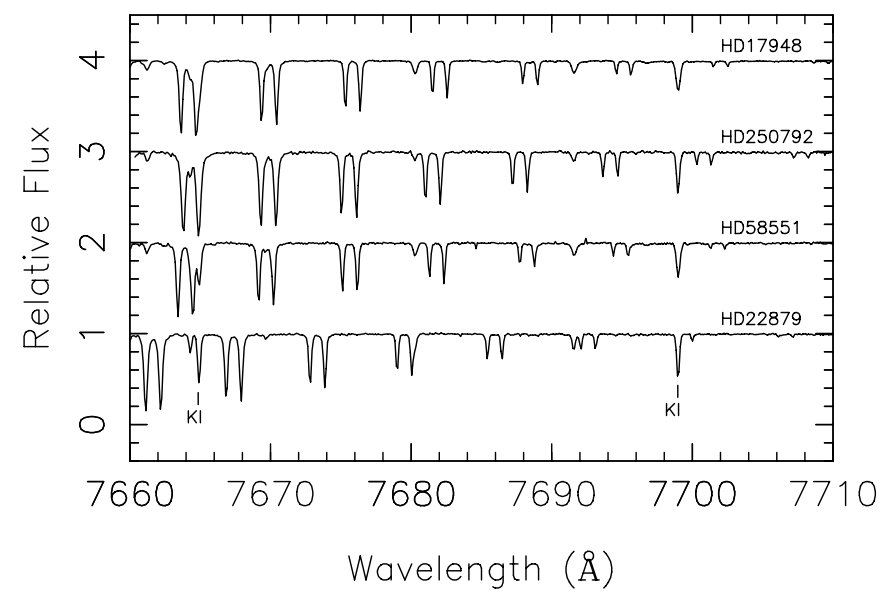

Fig. 1. Examples of normalized spectra in the region of the K I lines. The spectra were shifted vertically by 1.0 units in relative flux. The K I line at $7664.92 \AA$ is blended with the very strong telluric $\mathrm{O}_{2}$ lines in some cases.

\section{Observational data}

The high-resolution spectra analyzed in our present investigation were obtained with the FOCES fiber optics echelle spectrograph fed by the $2.2 \mathrm{~m}$ telescope of the DSAZ at Calar Alto Observatory during August 2001 and January 2003. The observations cover a spectral range from 3700 to $9800 \AA$ in a total of 97 spectral orders. The spectra were exposed on a $2048^{2}$ CCD chip with $24 \mu \mathrm{m}$ pixel size, providing a spectral resolution of $R \sim 40000$ per 2 pixel resolution element. All stars were observed at least twice with $S / N$ per pixel $\sim 100$ to 200 (see Gehren et al. 2004, 2006, for details). Data extraction followed the standard automatic IDL program environment designed for the FOCES spectrograph (Pfeiffer et al. 1998).

Examples of normalized spectra at regions of KI lines are given in Fig. 1. It should be kept in mind that the $\mathrm{K} I$ line at $7664.92 \AA$ is blended with the very strong telluric $\mathrm{O}_{2}$ lines in some cases because of various topocentric radial velocities.

\section{Atmospheric models and stellar parameters}

Stellar atmospheric models have been calculated for the individual stars using plane-parallel one-dimensional stratifications of temperature and pressure. We use line-blanketed LTE model atmospheres, generated as discussed by Fuhrmann et al. (1997). The mixing-length parameter $l / H_{\mathrm{p}}$ is adopted to be 0.5 (Fuhrmann et al. 1993). The ODF data are provided by Kurucz (1992); they are rescaled by $\Delta[\mathrm{Fe} / \mathrm{H}]=-0.16$ to match the meteoritic iron abundance of Anders \& Grevesse (1989), $\log \varepsilon_{\odot}(\mathrm{Fe})=7.51$.

We adopt the stellar parameters for the sample stars determined by Gehren et al. (2004, 2006). Effective temperatures are derived from fitting the wings of the Balmer line $\mathrm{H}_{\alpha}$ and $\mathrm{H}_{\beta}$ profiles. The surface gravities are calculated from Hipparcos parallaxes (ESA 1997). Iron abundances are based on Fe II lines, and the microturbulence velocities are estimated by demanding that the iron abundance derived from Fe II lines should not depend on equivalent widths. Gehren et al. $(2004,2006)$ estimated the uncertainties for the temperature, surface gravity, metal abundance, and microturbulence velocity for most of the stars as $\pm 80 \mathrm{~K}$, 0.10 dex, 0.05 dex and $0.2 \mathrm{~km} \mathrm{~s}^{-1}$, respectively.

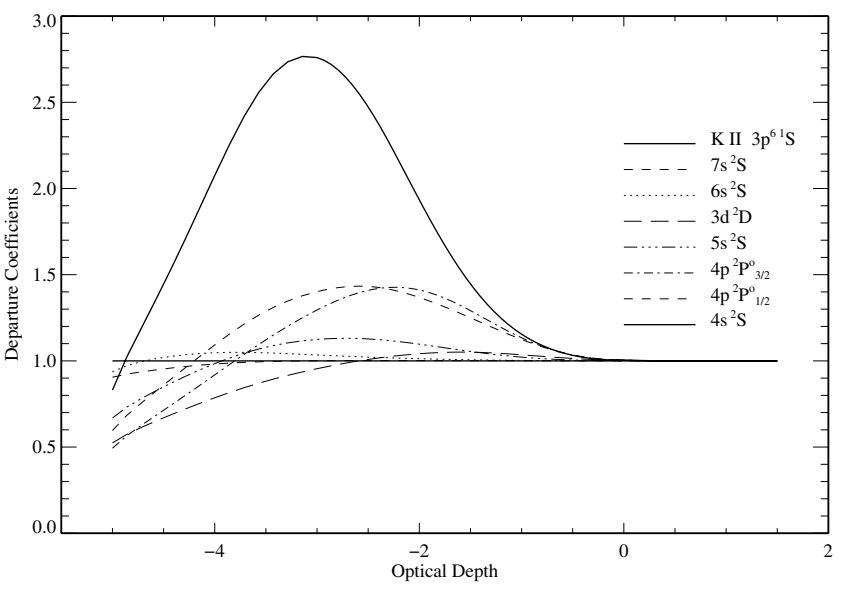

Fig. 2. Typical example of collision-dominated K I population departure coefficients $b_{n}=N_{n}^{\mathrm{NLTE}} / N_{n}^{\mathrm{LTE}}$ in the moderately metal-poor star HD 58551. The kinetic equilibrium calculations are described in the text.

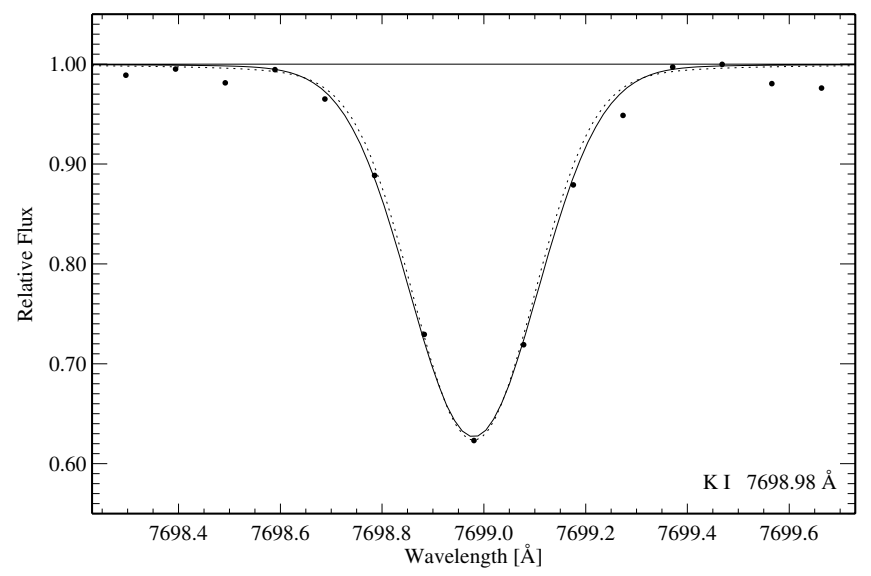

Fig. 3. Synthetic flux profiles under LTE (dotted line) and NLTE (solid line) conditions compared with the observed spectra (filled circles) of HD 58551.

\section{Abundance analysis}

\subsection{Atomic models}

All calculations have been carried out with a revised version of the DETAIL program (Butler \& Giddings 1985) using the accelerated lambda iteration (see Gehren et al. 2001, 2004 for details).

Our investigation is based on the atomic properties documented in Paper I. The total number of levels was 67 plus the ground state of K II. Oscillator strengths and photoionization cross-sections have been calculated by Butler (2000, private communication). Hydrogen collisions are supplied with the standard enhancement factor $S_{\mathrm{H}}$ and the full analysis of the solar spectrum allows a reasonable choice of $S_{\mathrm{H}}=0.05$ for K I. A typical example of collision-dominated departure coefficients is given in Fig. 2.

\subsection{Spectrum synthesis}

The abundance determinations for our sample stars are made using the spectrum synthesis program SIU, which is an IDL/Fortran-based software package (Reetz 1993, private communication). The synthetic spectra are convolved with 
Table 1. Atomic data of K I lines.

\begin{tabular}{ccccc}
\hline \hline$\lambda[\AA]]$ & $E_{\text {low }}[e v]$ & Transition & $\log g f \varepsilon_{\odot}$ & $\log C_{6}$ \\
\hline 7664.92 & 0.00 & $4 \mathrm{~s}^{2} \mathrm{~S}_{1 / 2}-4 \mathrm{p}^{2} \mathrm{P}_{3 / 2}^{\mathrm{o}}$ & 5.27 & -31.00 \\
7698.98 & 0.00 & $4 \mathrm{~s}^{2} \mathrm{~S}_{1 / 2}-4 \mathrm{p}^{2} \mathrm{P}_{1 / 2}^{\mathrm{o}}$ & 4.97 & -31.00 \\
\hline
\end{tabular}

macroturbulence, rotational and instrumental broadening profiles in order to match the observed spectral lines.

Based on the 7665/7699 line pair, our abundance results are obtained from profile fits under both LTE and NLTE conditions. The atomic data of the KI lines are listed in Table 1, in which the oscillator strengths are calculated by Butler (2000, private communication). The van der Waals damping constants are determined by fitting the solar resonance line wings. This requires an enhancement of 0.11 dex over the values calculated from Anstee \& O'Mara's (1995) tables (see Paper I for details). Since our results are based on a differential analysis with respect to the Sun, Table 1 lists the relevant line data with reference to the solar potassium abundance (Paper I).

In order to avoid the effect of blends with telluric lines, the spectra of all sample stars were carefully checked, all potassium lines with blends were excluded.

The stellar parameters taken from Gehren et al. $(2004,2006)$, the potassium abundances for individual lines, and average results of 58 sample stars are presented in Table 2. Our results show good agreement between the 7665 and 7699 lines. The NLTE abundance scatter of the two lines is between 0.00 and 0.07 dex in individual stars with a mean value of $\langle\sigma[\mathrm{K} / \mathrm{Fe}]\rangle=$ $0.008 \pm 0.006$ for all 23 stars with both lines available.

\subsection{Comparison with other NLTE work}

Potassium abundances of metal-poor stars have been determined recently by Takeda et al. (2002) and Shimansky et al. (2003), both based on NLTE analysis. We find two stars (HD 60319 and HD 208906) in our sample in common with Shimansky et al. (2003). The agreement is good and differences between the NLTE abundances are 0.04 and 0.08 dex. Six stars are in common with Takeda et al. (2002), for which three stars (HD 60319, HD 208906 and HD 224930) show good agreement; the differences are less than 0.04 dex. The other three stars (HD 58551, HD 59984 and HD 134169) show a very large abundance difference $(\sim 0.20 \mathrm{dex})$. We note that this abundance difference is mostly due to the different stellar parameters adopted; changing the effective temperature by $200 \mathrm{~K}$ will produce $\sim 0.15 \mathrm{dex}$ abundance difference. Our calculations show that the difference of abundance results would be less than $0.10 \mathrm{dex}$, if the stellar parameters of Takeda et al. (2002) were adopted.

\section{Discussion}

\subsection{NLTE effects}

Our results demonstrate how much the potassium abundances of metal-poor stars depend on the assumption of the thermodynamic properties in stellar atmospheres. Since only the resonance lines can be observed, there is no simple observational evidence that rules out LTE in our stellar sample. However, the thermodynamic conditions in metal-poor stars are much less favorable for LTE than in the Sun, which can be shown to follow NLTE predictions. The variation of the NLTE corrections for our sample stars, $\Delta[\mathrm{K} / \mathrm{Fe}]=[\mathrm{K} / \mathrm{Fe}]_{\mathrm{NLTE}}-[\mathrm{K} / \mathrm{Fe}]_{\mathrm{LTE}}$, on effective temperature, gravity and metal abundance given in Fig. 4 leads to the following conclusions:

1. The NLTE corrections for all stars are negative and lie in the range -0.20 to $-0.55 \mathrm{dex}$, with an average of $-0.40 \mathrm{dex}$.

2. The NLTE effect increases with increasing effective temperature.

3. The NLTE effect increases with decreasing gravity, and

4. The NLTE corrections are independent of metal abundance for $[\mathrm{Fe} / \mathrm{H}] \geq-1.2$.

The tendency that the NLTE effects in potassium are large for warm subgiant stars is similar to the sodium NLTE studies (e.g. Shi et al. 2004). As expected, stronger departures from LTE are found for stars with high temperature, which is in agreement with the statistical equilibrium of sodium, magnesium and aluminium (Gehren et al. 2004, 2006). The reduction of surface gravity results in a decreased efficiency of collisions with electrons and hydrogen atoms, which again leads to stronger NLTE effects. We do not find dependencies of $\Delta[\mathrm{K} / \mathrm{Fe}]$ on the metal abundance with $[\mathrm{Fe} / \mathrm{H}] \geq-1.2$, however, as shown in Fig. 7 of Gehren et al. (2006), such trends are likely to appear as the metal abundance decreases below $[\mathrm{Fe} / \mathrm{H}]=-1.5$.

\section{2. $[\mathrm{K} / \mathrm{Fe}]$ versus $[\mathrm{Fe} / \mathrm{H}]$}

Figure 5 shows the run of the $[\mathrm{K} / \mathrm{Fe}]$ ratio (calculated in NLTE) with metal abundance for all stars considered in this paper. A tentative error estimate has to account for the errors of the adopted stellar parameters. Based on the errors of stellar parameters estimated in Gehren et al. (2004, 2006), the corresponding maximum rms error would be \pm 0.09 . As usual the error due to the quality of the spectra is small and can be neglected for most of the KI resonance lines. It appears in the abundance differences found between the two resonance lines and averages around 0.02 dex, with the exception of HD 97855A, HD 158226, HD 215257, and HD 221876. Taking into account the full error budget, the average abundance error is estimated to be $\simeq 0.04 \ldots 0.06$ dex. However, this estimate does not account for systematic errors due to the disadvantages of our atmospheric models (1D structure, ODFs etc.). Much of the scatter seen in Figs. 4 and 5 must be real; in particular the separation between thin and thick disk stars in Fig. 5 cannot be explained by errors in the analysis.

The identification of population membership of individual stars is difficult. Kinematic data alone do not allow the unambiguous identification of a stellar population. In particular the overlap between the different populations has not yet been fully evaluated. Gehren et al. (2006) discussed the redefinition of population membership based on abundances, kinematics, and ages. In this paper we use the criteria of Gehren et al. (2006) to separate halo, thin and thick disks stars and plot them using different symbols (see caption of Fig. 5).

In Fig. 5, we can see that $[\mathrm{K} / \mathrm{Fe}]$ gradually increases with a decrease of $[\mathrm{Fe} / \mathrm{H}]$ over the range of $-0.8 \leq[\mathrm{Fe} / \mathrm{H}] \leq 0.0$ for thin disk stars. For thick disk stars, $[\mathrm{K} / \mathrm{Fe}]$ is nearly constant over the range of $-1.0 \leq[\mathrm{Fe} / \mathrm{H}] \leq-0.4$, the average $[\mathrm{K} / \mathrm{Fe}]$ of 20 thick disk stars is $+0.27 \pm 0.07$ dex. The fact that the potassium abundance of thin and thick disk stars shows different abundance trends is very similar to the magnesium result of Fuhrmann (1998) and the $\alpha$-elements (Mg, Si, Ca and Ti) of Bensby et al. (2003, 2005). Such a behaviour also supports the assumption that thin and thick disks formed at epochs that are clearly separated in time and/or space. 
Table 2. Stellar parameters and potassium abundances of the sample stars.

\begin{tabular}{|c|c|c|c|c|c|c|c|c|c|c|}
\hline \multirow[t]{2}{*}{ Star } & \multirow{2}{*}{$\begin{array}{l}T_{\text {eff }} \\
(\mathrm{K}) \\
\end{array}$} & \multirow[t]{2}{*}{$\log g$} & \multirow[t]{2}{*}[\mathrm{Fe}/\mathrm{H}]{} & \multirow{2}{*}{$\begin{array}{c}\xi_{\mathrm{t}} \\
\left(\mathrm{km} \mathrm{s}^{-1}\right) \\
\end{array}$} & \multicolumn{2}{|c|}{$\begin{array}{c}\mathrm{K} / \mathrm{Fe}] \\
7699\end{array}$} & \multicolumn{2}{|c|}{$\begin{array}{c}\mathrm{K} / \mathrm{Fe}] \\
7665\end{array}$} & \multicolumn{2}{|c|}{$\begin{array}{c}\mathrm{K} / \mathrm{Fe}] \\
\text { Average }\end{array}$} \\
\hline & & & & & LTE & NLTE & LTE & NLTE & LTE & NLTE \\
\hline HD 224930 & 5480 & 4.45 & -0.66 & 0.9 & +0.60 & +0.28 & +0.55 & +0.29 & +0.58 & +0.29 \\
\hline G69-8 & 5640 & 4.43 & -0.55 & 1.1 & +0.64 & +0.25 & +0.60 & +0.25 & +0.62 & +0.25 \\
\hline HD 17948 & 6325 & 4.13 & -0.35 & 1.9 & +0.50 & -0.02 & - & - & +0.50 & -0.02 \\
\hline HD 22309 & 5900 & 4.29 & -0.31 & 1.3 & +0.40 & -0.02 & - & - & +0.40 & -0.02 \\
\hline HD 22879 & 5775 & 4.26 & -0.83 & 1.1 & - & - & +0.65 & +0.22 & +0.65 & +0.22 \\
\hline HD 241253 & 5850 & 4.25 & -1.05 & 1.2 & +0.60 & +0.18 & - & - & +0.60 & +0.18 \\
\hline HD 243357 & 5675 & 4.38 & -0.59 & 1.1 & +0.60 & +0.29 & - & - & +0.60 & +0.29 \\
\hline HD 36283 & 5475 & 4.28 & -0.41 & 0.8 & +0.60 & +0.31 & +0.60 & +0.34 & +0.60 & +0.33 \\
\hline G99-21 & 5525 & 4.30 & -0.63 & 1.0 & +0.60 & +0.29 & - & - & +0.60 & +0.29 \\
\hline HD 250792 & 5600 & 4.32 & -1.02 & 1.1 & +0.65 & +0.30 & - & - & +0.65 & +0.30 \\
\hline HD 56513 & 5630 & 4.53 & -0.45 & 1.2 & +0.33 & +0.04 & +0.28 & +0.07 & +0.31 & +0.06 \\
\hline HD 58551 & 6190 & 4.23 & -0.53 & 1.8 & +0.55 & +0.05 & - & - & +0.55 & +0.05 \\
\hline HD 59374 & 5840 & 4.37 & -0.83 & 1.4 & +0.66 & +0.19 & +0.58 & +0.18 & +0.62 & +0.19 \\
\hline HD 59984 & 5925 & 3.94 & -0.74 & 1.2 & +0.75 & +0.22 & - & - & +0.75 & +0.22 \\
\hline HD 60319 & 5875 & 4.17 & -0.82 & 1.4 & +0.68 & +0.25 & +0.70 & +0.28 & +0.69 & +0.27 \\
\hline HD 233511 & 6015 & 4.29 & -1.57 & 1.4 & +0.46 & +0.24 & - & - & +0.46 & +0.24 \\
\hline G235-45 & 5500 & 4.25 & -0.59 & 1.1 & +0.75 & +0.40 & - & - & +0.75 & +0.40 \\
\hline HD 88446 & 5915 & 4.03 & -0.44 & 1.6 & +0.55 & +0.00 & - & - & +0.55 & +0.00 \\
\hline HD 88725 & 5665 & 4.35 & -0.70 & 1.2 & +0.55 & +0.25 & +0.55 & +0.24 & +0.55 & +0.25 \\
\hline HD 91784 & 5890 & 4.47 & -0.33 & 1.3 & +0.55 & +0.22 & - & - & +0.55 & +0.22 \\
\hline G119-32 & 5715 & 4.39 & -1.88 & 1.2 & +0.42 & +0.21 & - & - & +0.42 & +0.21 \\
\hline HD 96094 & 5900 & 4.01 & -0.46 & 1.7 & +0.58 & +0.05 & - & - & +0.58 & +0.05 \\
\hline HD 97855A & 6240 & 4.13 & -0.44 & 1.8 & +0.60 & +0.07 & +0.58 & +0.00 & +0.59 & +0.04 \\
\hline $\mathrm{BD}+20^{\circ} 2594$ & 5900 & 4.30 & -0.81 & 1.4 & +0.36 & -0.06 & - & - & +0.36 & -0.06 \\
\hline HD 104056 & 5875 & 4.31 & -0.41 & 1.3 & +0.50 & +0.10 & +0.50 & +0.12 & +0.50 & +0.11 \\
\hline HD 108076 & 5725 & 4.44 & -0.73 & 1.2 & +0.50 & +0.17 & +0.50 & +0.17 & +0.50 & +0.17 \\
\hline HD 114606 & 5610 & 4.28 & -0.57 & 1.2 & +0.65 & +0.35 & - & - & +0.65 & +0.35 \\
\hline HD 118659 & 5510 & 4.36 & -0.60 & 1.0 & +0.60 & +0.40 & +0.55 & +0.36 & +0.58 & +0.38 \\
\hline HD 119288 & 6420 & 4.13 & -0.17 & 1.9 & +0.35 & -0.12 & - & - & +0.35 & -0.12 \\
\hline HD 123710 & 5790 & 4.41 & -0.54 & 1.4 & +0.60 & +0.21 & - & - & +0.60 & +0.21 \\
\hline HD 126512 & 5825 & 4.02 & -0.64 & 1.6 & +0.70 & +0.25 & +0.70 & +0.22 & +0.70 & +0.24 \\
\hline HD 134169 & 5930 & 3.98 & -0.86 & 1.8 & +0.80 & +0.42 & - & - & +0.80 & +0.42 \\
\hline HD 142267 & 5807 & 4.42 & -0.46 & 1.0 & +0.58 & +0.15 & - & - & +0.58 & +0.15 \\
\hline HD 144061 & 5815 & 4.44 & -0.31 & 1.2 & +0.50 & +0.10 & - & - & +0.50 & +0.10 \\
\hline HD 148816 & 5880 & 4.07 & -0.78 & 1.2 & +0.75 & +0.24 & - & - & +0.75 & +0.24 \\
\hline HD 149996 & 5665 & 4.09 & -0.52 & 1.2 & +0.60 & +0.28 & +0.60 & +0.26 & +0.60 & +0.27 \\
\hline $\mathrm{BD}+68^{\circ} 901$ & 5715 & 4.51 & -0.25 & 1.4 & +0.35 & +0.00 & - & - & +0.35 & +0.00 \\
\hline HD 157089 & 5800 & 4.06 & -0.59 & 1.2 & +0.63 & +0.16 & +0.65 & +0.19 & +0.64 & +0.18 \\
\hline HD 157466 & 5990 & 4.38 & -0.44 & 1.1 & +0.45 & +0.02 & - & - & +0.45 & +0.02 \\
\hline HD 158226 & 5805 & 4.12 & -0.56 & 1.1 & +0.82 & +0.34 & +0.78 & +0.29 & +0.80 & +0.32 \\
\hline G170-56 & 6030 & 4.31 & -0.79 & 1.3 & +0.44 & -0.02 & - & - & +0.44 & -0.02 \\
\hline HD 160933 & 5765 & 3.85 & -0.27 & 1.2 & +0.45 & -0.08 & +0.45 & -0.08 & +0.45 & -0.08 \\
\hline HD 160693 & 5850 & 4.31 & -0.60 & 1.2 & +0.65 & +0.19 & - & - & +0.65 & +0.19 \\
\hline HD 170357 & 5665 & 4.07 & -0.50 & 1.2 & +0.60 & +0.35 & - & - & +0.60 & +0.35 \\
\hline HD 171620 & 6115 & 4.20 & -0.50 & 1.4 & +0.60 & +0.13 & +0.60 & +0.15 & +0.60 & +0.14 \\
\hline G142-2 & 5675 & 4.48 & -0.75 & 1.1 & +0.55 & +0.25 & - & - & +0.55 & +0.25 \\
\hline HD 182807 & 6100 & 4.21 & -0.33 & 1.4 & +0.52 & +0.06 & - & - & +0.52 & +0.06 \\
\hline HD 184448 & 5765 & 4.16 & -0.43 & 1.2 & +0.65 & +0.27 & - & - & +0.65 & +0.27 \\
\hline HD 186379 & 5865 & 3.93 & -0.41 & 1.2 & +0.48 & +0.02 & - & - & +0.48 & +0.02 \\
\hline HD 198300 & 5890 & 4.31 & -0.60 & 1.2 & +0.65 & +0.31 & +0.63 & +0.31 & +0.64 & +0.31 \\
\hline HD 200580 & 5940 & 3.96 & -0.82 & 1.4 & +0.85 & +0.33 & - & - & +0.85 & +0.33 \\
\hline HD 204155 & 5815 & 4.09 & -0.66 & 1.2 & - & - & +0.58 & +0.17 & +0.58 & +0.17 \\
\hline G188-22 & 6040 & 4.37 & -1.25 & 1.5 & +0.48 & +0.20 & +0.50 & +0.16 & +0.49 & +0.18 \\
\hline HD 208906 & 6025 & 4.37 & -0.76 & 1.4 & +0.55 & +0.09 & - & - & +0.55 & +0.09 \\
\hline G242-4 & 5815 & 4.31 & -1.10 & 1.2 & +0.60 & +0.16 & +0.60 & +0.16 & +0.60 & +0.16 \\
\hline HD 215257 & 6030 & 4.28 & -0.58 & 1.4 & +0.60 & +0.20 & +0.60 & +0.14 & +0.60 & +0.17 \\
\hline HD 218209 & 5665 & 4.40 & -0.60 & 1.1 & +0.65 & +0.30 & +0.65 & +0.33 & +0.65 & +0.32 \\
\hline HD 221876 & 5865 & 4.29 & -0.60 & 1.2 & +0.50 & +0.14 & +0.50 & +0.08 & +0.50 & +0.11 \\
\hline
\end{tabular}

Figure 5 also shows that the halo stars have nearly constant values of $[\mathrm{K} / \mathrm{Fe}] \simeq+0.20$ dex. We note that there are two outliers: G170-56 and $\mathrm{BD}+20^{\circ} 2594$, with a significantly lower $[\mathrm{K} / \mathrm{Fe}] \mathrm{ra}-$ tio. Gehren et al. $(2004,2006)$ also showed a solar $[\mathrm{Mg} / \mathrm{Fe}]$ ratio and significantly underabundant values for $\mathrm{Na}$ and $\mathrm{Al}$ in these two stars. They claimed that these stars may have followed some peculiar history and could have been accreted from dwarf galaxies or star clusters. 

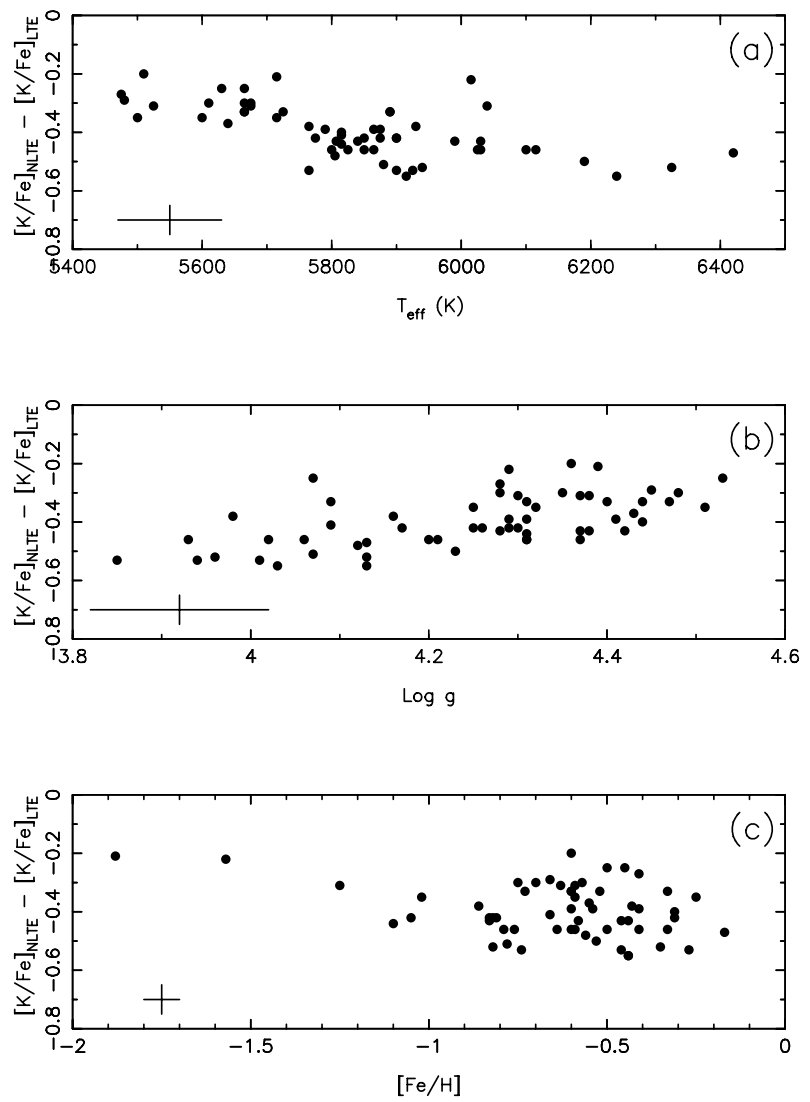

Fig. 4. Dependences of NLTE corrections for our sample stars on a) effective temperature, b) gravity and c) metal abundance.

Our sample includes only 2 very metal-poor stars with $[\mathrm{Fe} / \mathrm{H}]<-1.5$. Some works presented potassium abundances of more very metal-poor stars, although there might be systematic differences due to the different analytical methods. Based on a NLTE analysis, Takeda et al. (2002) found that [K/Fe] appears to be nearly flat with $\sim 0.3$ to $0.4 \mathrm{dex}$ (or slightly increasing) in the region of $-2.4 \leq[\mathrm{Fe} / \mathrm{H}] \leq-1.0$. Cayrel et al. (2004) presented potassium abundances for a large sample of very metalpoor giant stars with $[\mathrm{Fe} / \mathrm{H}]<-2.5$ and found that $[\mathrm{K} / \mathrm{Fe}]$ seems to decrease slowly with decreasing metal abundance, although the slope is not very significant. We note that their results were derived from an LTE analysis with similar NLTE corrections for all sample stars taken from Ivanova \& Shimansky (2000) because of the narrow range of stellar parameters covered by their sample, but the NLTE corrections are not only a matter of stellar parameters. Since they also depend on the potassium abundance itself, individual corrections treated in such a global way will therefore produce additional abundance scatter, which may hide properties of the abundance ratios (Gehren et al. 2004, 2006). Hill et al. (2002) found a potassium abundance of CS 31082-001 with $[\mathrm{Fe} / \mathrm{H}]=-2.9$ which would be around $[\mathrm{K} / \mathrm{Fe}]=+0.3 \mathrm{dex}$ after the NLTE correction. Depagne et al. (2002) find that the $\mathrm{K}$ doublet is undetected in the spectra of both CS 22949-037 and $\mathrm{CD}-38245$ with $[\mathrm{Fe} / \mathrm{H}] \sim-4.0$, corresponding to an upper limit of $[\mathrm{K} / \mathrm{Fe}] \leq-0.1$.

\subsection{Potassium abundance and nucleosynthesis in the Galaxy}

The variation of $[\mathrm{K} / \mathrm{Fe}]$ with the stellar metal abundance contains information about the chemical evolution of the Galaxy. In Fig. 5

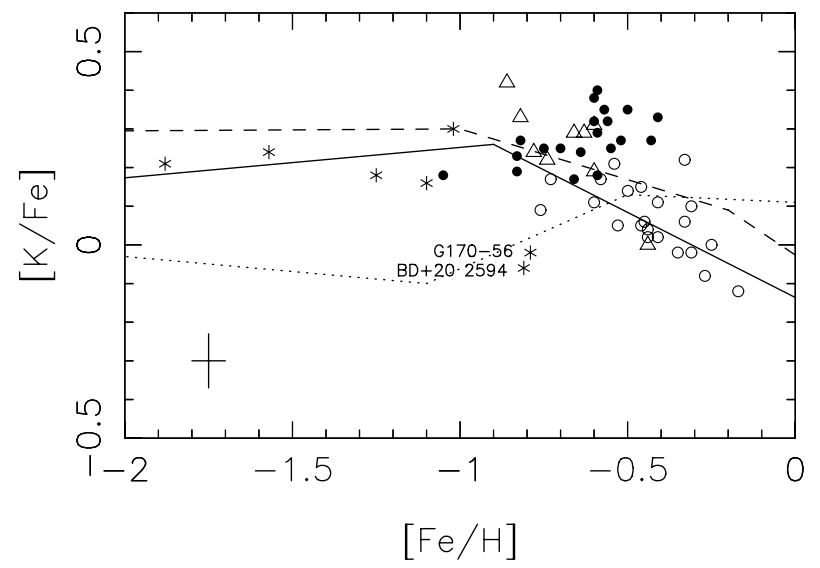

Fig. 5. Abundance ratios $[\mathrm{K} / \mathrm{Fe}]$ for NLTE analysis. Open circles refer to the thin-disk stars, filled circles to the thick-disk stars, asterisks to the halo stars, open triangle to the stars with uncertain population membership. The solid line shows the theoretical predictions of Goswami \& Prantzos (2000), the dashed line is the one of Samland (1998), and the dotted line refers to Timmes et al. (1995).

theoretical models on the chemical evolution of potassium are also given.

As noticed in previous studies (Takeda et al. 2002; Shimansky et al. 2003), the model of Timmes et al. (1995) based on Woosley \& Weaver's (1995) yields and Salpeter's (1955) Initial Mass Function (IMF) apparently contradicts the supersolar $[\mathrm{K} / \mathrm{Fe}]$ trend in metal-poor stars. Our observational data of thin disk and halo stars are consistent with the theoretical prediction of Goswami \& Prantzos (2000) who used Kroupa et al.'s (1993) IMF. They are also well represented by potassium synthesis in a chemodynamical model as proposed by Samland et al. (1998). We also note that the flat $[\mathrm{K} / \mathrm{Fe}]$ trend of the thick disk stars is not predicted by existing theoretical models. In order to understand the abundance trend of thick disk stars a detailed consideration of how the thick disk population was formed is needed.

Using magnesium instead of iron as a reference, type Ia $\mathrm{SNe}$ can be removed from consideration. The overall behaviour of $[\mathrm{K} / \mathrm{Mg}]$ ratios vs. $[\mathrm{Fe} / \mathrm{H}],[\mathrm{Mg} / \mathrm{H}]$, and $[\mathrm{Mg} / \mathrm{Fe}]$ is shown in Figs. 6a, b, and c, where the $\mathrm{Mg}$ abundances are taken from Gehren et al. (2004, 2006).

We note that $[\mathrm{K} / \mathrm{Mg}]$ of our sample stars is nearly constant, with a small scatter of only 0.07 dex. The mean $[\mathrm{K} / \mathrm{Mg}]$ of 58 sample stars is $-0.08 \pm 0.01 \mathrm{dex}$, although two stars with $[\mathrm{Fe} / \mathrm{H}] \leq-1.5($ and $[\mathrm{Mg} / \mathrm{H}] \leq-1.0)$ have a lower $[\mathrm{K} / \mathrm{Mg}]$ value than the average. This behaviour needs confirmation from a study of very metal-poor stars with $[\mathrm{Fe} / \mathrm{H}]<-1.5$. Figure $6 \mathrm{c}$ seems to display a slightly decrease of $[\mathrm{K} / \mathrm{Mg}]$ for thick disk and halo stars with increasing $[\mathrm{Mg} / \mathrm{Fe}]$, but when the uncertainties of abundance determinations are considered, the trend is not significant.

The nearly constant $[\mathrm{K} / \mathrm{Mg}]$ ratio with small scatter in all metal abundance ranges suggests that the nucleosynthesis of potassium is closely coupled to that of the $\alpha$-elements. Whereas Gehren et al. (2006) find that the relation between $[\mathrm{Na} / \mathrm{Mg}]$ and $[\mathrm{Fe} / \mathrm{H}]$ enrichment is continuous through all three Galactic populations spanning a range of values between a metal-poor plateau at $[\mathrm{Na} / \mathrm{Mg}]=-0.7$ and solar values, $[\mathrm{Al} / \mathrm{Mg}]$ instead displays a step-like change between stars of the Galactic halo where $[\mathrm{Al} / \mathrm{Mg}] \sim-0.45$, and the two disk populations for which $[\mathrm{Al} / \mathrm{Mg}] \sim+0.10$. 

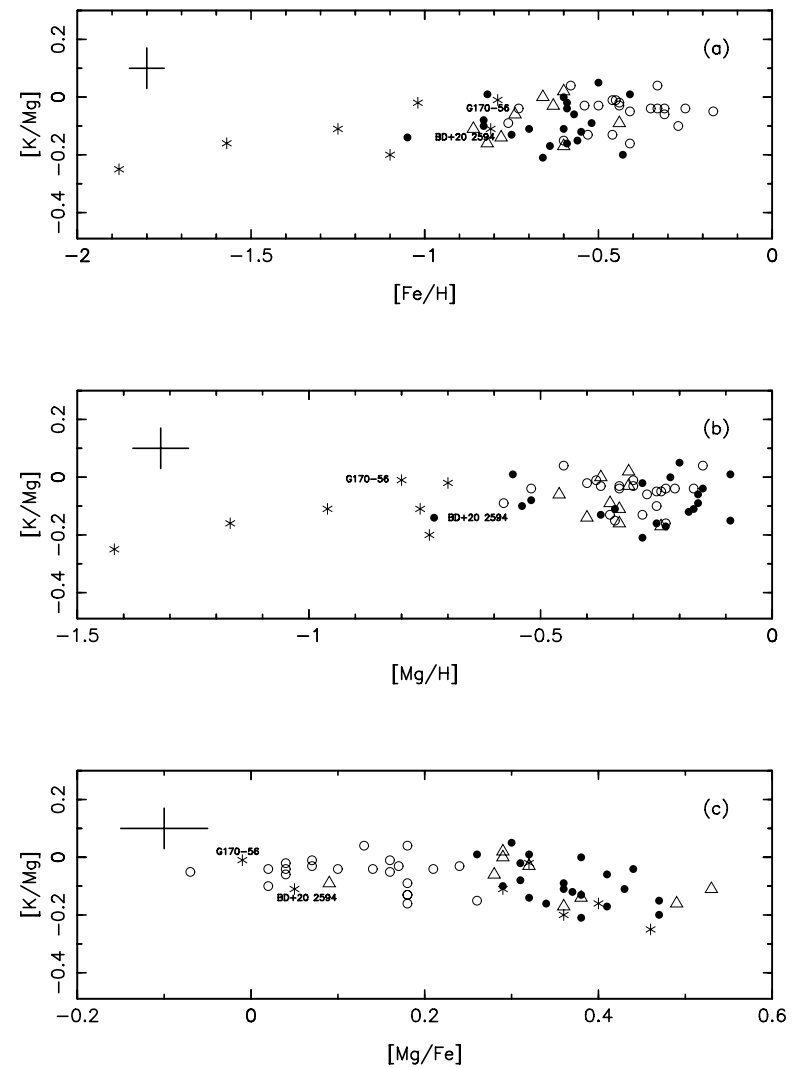

Fig. 6. $[\mathrm{K} / \mathrm{Mg}]$ abundances ratios from NLTE analyses as functions of $[\mathrm{Fe} / \mathrm{H}],[\mathrm{Mg} / \mathrm{H}]$, and $[\mathrm{Mg} / \mathrm{Fe}]$. Symbols are the same as in Fig. 5 .

Potassium, sodium, and aluminum are odd-Z elements mainly produced in massive stars. As their synthesis requires neutron capture reactions, the yields are affected by the initial metal abundance of the stars and, together with the $\alpha$-elements, they are expected to follow an odd-even effect. This has been discussed by Samland (1998), who remarked that the yields of odd elements in Woosley \& Weaver (1995) are slightly low but in rough agreement with the solar chemical composition. Although Timmes et al. (1995) have used these data, their high-mass stellar yields are obviously very different from those used by Samland. The reason for that difference is not immediately evident. It is buried in the initial mass functions, the use of different yields, but mainly in the ratio of Supernova explosions of types II and Ia.

Therefore, comparison of the abundance trends of potassium, sodium, and aluminium does not present direct information about their nucleosynthesis sites and processes. It also requires information on star formation rates and initial mass functions to understand why similar results of explosive nucleosynthesis can result in so different chemical evolution scenarios.

\section{Conclusions}

We have determined potassium abundances for 58 metal-poor stars, spanning the range $-1.9<[\mathrm{Fe} / \mathrm{H}]<-0.2$. All abundances are derived from NLTE statistical equilibrium calculations and spectral synthesis. The results of our study lead to the following conclusions:

1. The NLTE corrections to the potassium abundances derived from the KI 7699 and $7665 \AA$ lines for all sample stars are negative and vary in the range of -0.20 to $-0.55 \mathrm{dex}$ depending on the effective temperature and surface gravity of the stars. Large departures from LTE appear for warm subgiant and turnoff stars.

2. The potassium abundances of halo, thin, and thick disk stars show a distinct trend, such as for the $\alpha$-elements: $[\mathrm{K} / \mathrm{Fe}]$ gradually increases with a decrease in $[\mathrm{Fe} / \mathrm{H}]$ for thin disk stars, $[\mathrm{K} / \mathrm{Fe}]$ of thick disk stars is nearly constant at $-1.0 \leq[\mathrm{Fe} / \mathrm{H}] \leq-0.4$, the average $[\mathrm{K} / \mathrm{Fe}]$ of 20 thick disk stars is $+0.27 \pm 0.07$ dex. The halo stars also have nearly constant values of $[\mathrm{K} / \mathrm{Fe}] \sim+0.20$ dex.

3 . The dependence of $[\mathrm{K} / \mathrm{Fe}]$ vs. $[\mathrm{Fe} / \mathrm{H}]$ is in agreement with the theoretical prediction of the Galactic chemical evolution models of Samland (1998) or Goswami \& Prantzos (2000), but at variance with the results of Timmes et al. (1995).

4. The halo, thin, and thick disks stars display a nearly constant $[\mathrm{K} / \mathrm{Mg}]$ ratio with small scatter, which suggests that the nucleosynthesis of potassium is closely coupled to that of the $\alpha$-elements.

Acknowledgements. This project was supported by the Deutsche Forschungsgemeinschaft (DFG) under contracts Ge 490/26-1 and 446 CHV 112, and by the National Natural Science Foundation of China under grants No. 10433010 and 10521001. Z.H.W. and S.J.R. thank the Institute of Astronomy and Astrophysics of Munich University for warm hospitality during a productive stay in 2004 .

\section{References}

Anders, E., \& Grevesse, N. 1989, Geochim. Cosmochim. Acta, 53, 197 Anstee, S. D., \& O'Mara, B. J. 1995, MNRAS, 276, 859

Arnett, W. D. 1996, Supernovae and Nucleosynthesis (Princeton, New Jersey: Princeton Univ. Press)

Butler, K., \& Giddings, J. 1985, Newsletter on the analysis of astronomical spectra No. 9, University of London

Bensby, T., Feltzing, S., \& Lundström, I. 2003, A\&A, 410, 527

Bensby, T., Feltzing, S., Lundström, I., et al. 2005, A\&A, 433, 185

Cayrel, R., Depagne, E., Spite, M., et al. 2004, A\&A, 416, 1117

Chen, Y. Q., Nissen, P. E., Zhao, G., et al. 2000, A\&AS, 141, 491

Depagne, E., Hill, V., Spite, M., et al. 2002, A\&A, 390, 187

ESA 1997, The Hipparcos and Tycho Catalogues, ESA SP-1200

Fuhrmann, K., Axer, M., \& Gehren, T. 1993, A\&A, 271, 451

Fuhrmann, K., Pfeiffer, M., Frank, C., et al. 1997, A\&A, 323, 909

Fuhrmann, K. 1998, A\&A, 338, 161

Gehren, T., Butler, K., Mashonkina, L., Reetz, J., \& Shi, J. R. 2001, A\&A, 366, 981

Gehren, T., Liang, Y. C., Shi, J. R., Zhang, H. W., \& Zhao, G. 2004, A\&A, 413, 1045

Gehren, T., Shi, J. R., Zhang, H. W., Zhao, G., \& Korn, A. 2006, A\&A, 451, 1065

Gratton, R. G., \& Sneden, C. 1987, A\&A, 178, 179

Grevesse, N., \& Sauval, A. J. 1998, Space Sci. Rev., 85, 161

Goswami, A., \& Prantzos, N. 2000, A\&A, 359, 191

Hill, V., Plez, R., Cayrel, R., et al. 2002, A\&A, 378, 560

Ivanova, D. V., \& Shimansky, V. V. 2000, Astr. Rep., 77, 432

Kroupa, P., Tout, C. A., \& Gilmore, G. 1993, MNRAS, 262, 545

Kurucz, R. L. 1992, Rev. Mex. Astron. Astrofis., 23, 45

Lodders, K. 2003, ApJ, 591, 1220

Pfeiffer, M., Frank, C., Baumüller, D., et al. 1998, A\&AS, 130, 381

Shi, J. R., Gehren, T., \& Zhao, G. 2004, A\&A, 423, 683

Shimansky, V. V., Bikmaev, I. F., Galeev, A. I., et al. 2003, Astr. Rep., 80, 816

Salpeter, E. E. 1955, ApJ, 121, 161

Samland, M. 1998, ApJ, 496,155

Takeda, Y., Kato, K., Watanabe, Y., et al. 1996, PASJ, 48, 511

Takeda, Y., Zhao, G., Chen, Y. Q., et al. 2002, PASJ, 54, 275

Thielemann, F.-K., \& Arnett, W. D. 1985, ApJ, 295, 589

Timmes, F. X., Woosley, S. E., \& Weaver, T. A. 1995, ApJS, 98, 617

Tomkin, J., Edvardsson, B., Lambert, D. L., et al. 1997, A\&A, 327, 587

Truran, J. W., \& Arnett, W. D. 1971, Ap\&SS, 11, 430

Woosley, S. E., \& Weaver, T. A. 1995, ApJS, 101, 181

Woosley, S. E., Arnett, W. D., \& Clayton, D. D. 1973, ApJS, 26, 231

Zhang, H. W., \& Zhao, G. 2005, MNRAS, 364, 712

Zhang, H. W., Butler, K., Gehren, T., Shi, J. R., \& Zhao, G. 2006, A\&A, 453, 723 (Paper I) 